\title{
Spontaneous Transection of the Superficial Femoral Artery with Devastating Outcomes: A Case Report
}

\author{
Thilina Gunawardena, Manujaya Godakandage, Sachith Abeywickrama, Rezni Cassim, and \\ Mandika Wijeyaratne \\ University Vascular Surgical Unit, National Hospital of Colombo, Colombo, Sri Lanka
}

\begin{abstract}
Non-traumatic, simultaneous pseudoaneurysms of the bilateral superficial femoral arteries (SFAs) are extremely rare. Spontaneous transection of the SFA is another unique pathology. Here, we present a patient with end stage kidney disease who was diagnosed with bilateral, simultaneous SFA pseudoaneurysms. He had a recent history of methicillin-sensitive Staphylococcus aureus septicemia; therefore, infection was suspected to be the main cause. Complete transection of the SFA was noted during the operative exploration of the symptomatic left side. Wide debridement and autologous vein bypass were performed via a clean route. Unfortunately, two months later, recurrent surgical site infection caused distal anastomotic rupture and fatal sepsis.
\end{abstract}

Key Words: False aneurysm, Femoral artery, Spontaneous rupture, Infected aneurysm
Received April 20, 2021

Revised June 16, 2021

Accepted June 25, 2021

Published on July 12, 2021

Corresponding author: Thilina Gunawardena University Vascular Surgical Unit, National Hospital of Colombo, Colombo, 01000, Sri Lanka

Tel: 94777030315

E-mail: thilinamg@gmail.com https://orcid.org/0000-0002-3867-2780

Copyright (c) 2021 The Korean Society for Vascular Surgery

This is an Open Access article distributed under the terms of the Creative Commons Attribution Non-Commercial License (http://creativecommons.org/licenses/by-nc/4.0) which permits unrestricted non-commercial use, distribution, and reproduction in any medium, provided the original work is properly cited.

Cite this article; Vasc Specialist Int 2021. https://doi.org/10.5758/vsi.210032

\section{INTRODUCTION}

The common femoral artery and the proximal superficial femoral artery (SFA) are common sites for pseudoaneurysms because these vessels are frequently punctured during endovascular catheterization and recreational drug use [1,2]. However, non-traumatic pseudoaneurysms of SFAs are relatively uncommon. Diagnosis of bilateral, non-traumatic, simultaneous SFA pseudoaneurysms is an extremely remote possibility. Here we present a patient who had this presentation as a complication of a central venous catheter associated bacteremia. During operative exploration on the left side, the SFA was found to be completely transected. This case report aims to highlight the rare pathological findings as well as the challenges faced during management of this patient.

Written informed consent was obtained from the relatives prior to the publication of the case report. This case report was exempt approval by the Ethics Committee at the
National Hospital of Colombo, Sri Lanka.

\section{CASE}

A 51-year-old male patient with type 2 diabetes mellitus complicated by end stage kidney disease (ESKD) presented with painful swelling of his left thigh for 1 week. Three months earlier, he had undergone emergency hemodialysis via a temporary central venous catheter inserted into the left internal jugular vein. After 10 days, he developed fever and purulent discharge from the catheter exit site. Pus and blood cultures were positive for methicillin-sensitive Staphylococcus aureus (MSSA). The infected catheter was removed, and the patient was treated with appropriate intravenous (IV) antibiotics for 2 weeks. The repeat blood culture after 7 days of antibiotic therapy yielded no bacterial growth. The current presentation was one and a half months after this episode. He denied a history of trauma to the thigh. No attempts had been made to cannulate the 


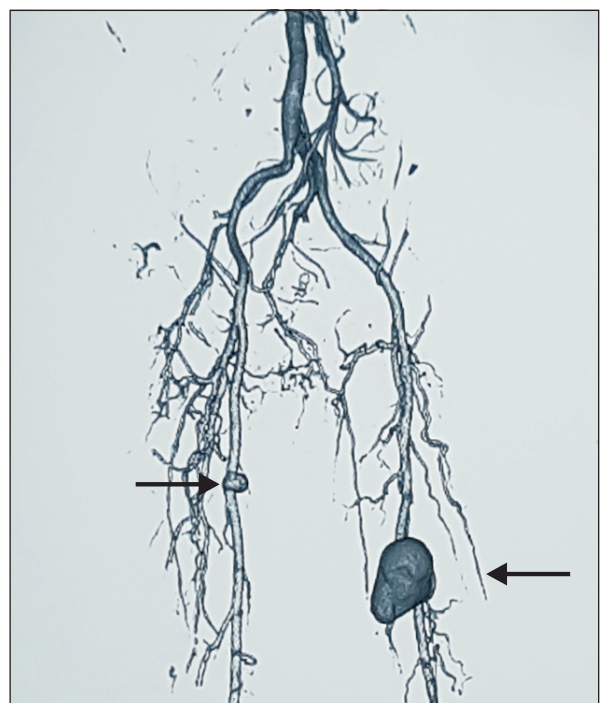

Fig. 1. Computed tomography image shows bilateral pseudoaneurysms in superficial femoral arteries (black arrows).

femoral vein for central venous access.

On admission, the patient was afebrile. The left thigh and leg were swollen. A tender, pulsatile bulge was noted on the medial aspect of the mid-thigh. Popliteal and pedal pulses were not felt on the affected side, but the foot was completely viable. His white blood cell count was 15,000/ $\mu \mathrm{L}$, with $82 \%$ neutrophils and the C-reactive protein level was $20.6 \mathrm{mg} / \mathrm{dL}$. A duplex ultrasound scan of the left thigh revealed a $4 \mathrm{~cm}$ pseudoaneurysm arising from middle segment of the left SFA. The adjacent segment of the femoral vein was thrombosed. Computed tomography angiography revealed bilateral SFA pseudoaneurysms (Fig. 1). The leftsided lesion was located $16 \mathrm{~cm}$ below the inguinal ligament, and measured $4.8 \mathrm{~cm} \times 6.2 \mathrm{~cm}$. On the right side, it was 10 $\mathrm{cm}$ below the inguinal ligament and measured $1.5 \mathrm{~cm} \times 1.5$ $\mathrm{cm}$. The two-dimensional echocardiogram was negative for infective endocarditis.

Infected pseudoaneurysms as a consequence of previous MSSA sepsis were suspected, and the patient was restarted on 6 hourly IV flucloxacillin at a dose of $500 \mathrm{mg}$. Repeated blood cultures were negative. We decided to operate on the symptomatic left pseudoaneurysm with pain and deep vein thrombosis (DVT). The SFA was explored proximal and distal to the lesion. The artery was extremely calcified. After administering 5,000 IU of IV heparin, the artery was clamped, and the pseudoaneurysm was opened. Surprisingly, the SFA was completely transected within the capsule of the pseudoaneurysm (Fig. 2). The vessel was ligated on either side of the rupture, and thorough debridement of the surrounding tissue was performed. The contralateral great saphenous vein (GSV) was harvested, reversed, and tun-



Fig. 2. The opened pseudoaneurysm cavity shows complete transection of the superficial femoral artery (proximal end, white arrow; distal end, black arrow).

neled lateral to the surgical field just beneath the deep fascia. Vein-to-SFA end-to-side anastomoses were performed proximally and distally to restore limb perfusion.

Histology of the pseudoaneurysm capsule revealed inflamed fibrous tissue. Bacteriological culture and special staining of debrided tissue failed to identify an organism. Postoperative recovery was complicated by the breakdown of the thigh wound. A decision was made to warfarinize the patient for 3 months for DVT and to conservatively follow up the pseudoaneurysm on the contralateral side for the time being. The patient was discharged on oral flucloxacillin for 3 months.

Two months later, the patient presented with massive bleeding from the left thigh. The prothrombin time international normalized ratio (INR) were within therapeutic limits. Anticoagulation was reversed, and re-exploration revealed dehiscence of the distal anastomosis of the bypass. The graft was ligated for hemostasis. Considering the physiological parameters at that time, we felt that he was not a candidate for a repeat bypass. The limb rapidly became ischemic. Despite extensive counseling, the patient refused amputation and died 2 days later.

\section{DISCUSSION}

A pseudoaneurysm occurs when focal damage to the wall of an artery is contained by the surrounding hematoma. Penetrating trauma is the leading cause of such lesions. Traumatic pseudoaneurysms frequently occur in the common femoral artery and proximal SFA because of arterial punctures performed during endovascular procedures and 
recreational drug use. Simultaneous, non-traumatic, infected pseudoaneurysms affecting bilateral SFAs, such as this case, have rarely been reported in the literature. Elzein et al. [3] described such a presentation in a patient with miliary tuberculosis. In contrast, unilateral infected pseudoaneurysm of the SFA is relatively common, and Staphylococcus aureus, Salmonella spp., and E.coli have been described as causative organisms [4-6]. As our patient had a history of MSSA sepsis, we suspected infection as the root cause of his presentation. However, blood and tissue cultures from the debrided pseudoaneurysm capsule failed to isolate an organism. This could be due to the antibiotics he received for the recent bacteremia.

Spontaneous transection of the SFA is rare. Spontaneous rupture of this vessel has been reported in patients with connective tissue disorders, atherosclerosis, and severe vessel wall calcification [7,8]. Our patient was diabetic and, had ESKD. Both these conditions are risk factors for accelerated atherosclerosis and vessel wall calcification. In fact, during surgery, we found that the SFA was heavily calcified. Possible bacterial seeding of the vessel wall or an atherosclerotic plaque may also have contributed to further weakening of the vessel wall.

The bulk of the literature on the management of infected pseudoaneurysm of the femoral artery is based on the experience derived from managing such lesions at the groin in IV drug abusers. Ligation of the artery above and below the pseudoaneurysm with debridement of the infected tissue is the most common management strategy performed in these cases. This is because of the prohibitive risk of graft infection and rupture when reconstruction is performed using prosthetic material. Native superficial or deep veins are unsuitable conduits in these patients because they tend to be thrombosed owing to previous drug injections $[2,9,10]$. Arterial ligation appears to be fairly well tolerated in this group, probably because of abundant collateral vessels that form as a response to the chronic arterial injury associated with repeated needle punctures [10]. Reconstruction using a vein patch, reversed saphenous vein graft bypass, endovascular stenting, and ligation of the artery have been used as options for managing proven or suspected infected pseudoaneurysms of the SFA that are not caused by IV drug abuse $[6,7,11,12]$. Covered stents deployed in a potentially infected field can be associated with stent infection and devastating outcomes [13]. In our case, we performed a reversed saphenous vein bypass graft to restore arterial continuity, as ligation of the SFA was not a viable option, as proven by the subsequent clinical events. To avoid traversing the graft through an infected field, we tunneled it laterally under the deep fascia of the thigh. Despite taking such precautions to avoid graft infection, our patient suffered disruption of the distal anastomosis, which was probably a consequence of infection secondary to his thigh wound breakdown.

SFA pseudoaneurysms, which are small, asymptomatic, and without pressure effects, can be managed conservatively [7]. However, this strategy may not be suitable for an infected pseudoaneurysm, and our patient would have eventually required intervention for the right-sided lesion. The decision to warfarinize the patient was based on current recommendations to treat provoked, proximal DVT with therapeutic anticoagulation for 3 months [14]. Considering the history, location of the bleeding, and the therapeutic INR level, immediate re-exploration was performed, suspecting secondary hemorrhage from the graft.

When the patient presented with bleeding, removal of the infected graft and a repeat bypass using an autologous vein would have been an option. Considering the DVT in the right femoral vein and the previous wound breakdown directly over the course of the right GSV, the ipsilateral GSV was not a suitable conduit. Alternative options would have been to harvest an arm vein or create a composite graft using the residual segment of the contralateral GSV. Both these procedures would have been time consuming, and at that time, we felt that the patient's physiology would not have permitted such an extensive procedure. Despite following the 'life before limb' principle, the patient unfortunately passed away due to multiple comorbidities and complications of an ischemic limb.

\section{CONFLICTS OF INTEREST}

The authors have nothing to disclose.

\section{ORCID}

Thilina Gunawardena
https://orcid.org/0000-0002-3867-2780
Manujaya Godakandage
https://orcid.org/0000-0002-1859-480X
Sachith Abeywickrama
https://orcid.org/0000-0002-8016-2258
Rezni Cassim
https://orcid.org/0000-0002-4826-6196
Mandika Wijeyaratne
https://orcid.org/0000-0001-9073-5446

\section{AUTHOR CONTRIBUTIONS}

Concept and design: TG. Analysis and interpretation: TG. Data collection: TG, MG, SA. Writing the article: TG, MG, SA. Critical revision of the article: RC, MW. Final ap- 
proval of the article: all authors. Statistical analysis: none. Obtained funding: none. Overall responsibility: TG.

\section{REFERENCES}

1) Rivera PA, Dattilo JB. Pseudoaneurysm [Internet]. Treasure 1sland: StatPearls Publishing; C 2021 [cited 2021 Mar 3]. Available from: https://www. ncbi.nlm.nih.gov/books/NBK542244/.

2) Levi N, Rørdam P, Jensen LP, Schroeder TV. Femoral pseudoaneurysms in drug addicts. Eur J Vasc Endovasc Surg 1997;13:361-362.

3) Elzein F, Qatan N, Alghamdi A, Albarrak $A$, Kalam K. Miliary tuberculosis presenting as bilateral superficial femoral artery mycotic aneurysm in an immunocompetent patient. Respir Med Case Rep 2019;26:236-239.

4) Carvalho PM, Mota JD, Dias PG, da Mota AO, de Moura JJ. Mycotic aneurysm of the femoral artery complicating Staphylococcus aureus bacteremia: a case report. Cases J 2009;2:9386.

5) Hussain M, Roche-Nagle G. Infected pseudoaneurysm of the superficial femoral artery in a patient with Salmonella enteritidis bacteremia. Can J Infect Dis Med Microbiol 2013;24:e24e25.
6) Ho MF, Chan YC, Cheng SW. Management of mycotic femoral artery aneurysm with two resistant organisms. Asian Cardiovasc Thorac Ann 2014;22:742-724.

7) Siani A, Flaishman I, Siani LM, Mounayergi F, Zaccaria A, Schioppa A, et al. Spontaneous rupture of the superficial femoral artery treated via an endovascular approach. Tex Heart Inst J 2008;35:66-68.

8) Cadier MA, Watkin G, Pope FM, Marston A. Spontaneous rupture of the femoral arteries. J R Soc Med 1993;86:54.

9) Salimi J, Shojaeefar A, Khashayar P. Management of infected femoral pseudoaneurysms in intravenous drug abusers: a review of 57 cases. Arch Med Res 2008;39:120-124.

10) Arora S, Weber MA, Fox CJ, Neville R, Lidor A, Sidawy AN. Common femoral artery ligation and local debridement: a safe treatment for infected femoral artery pseudoaneurysms. J Vasc Surg 2001;33:990-993.
11) Fukunaga N, Koyama T, Konoshi $Y$, Murashita T, Okada Y. Spontaneous rupture of superficial femoral artery. Ann Vasc Dis 2013;6:212-214.

12) Bowden DJ, Hayes PD, Sadat U, Choon See T. Mycotic pseudoaneurysm of the superficial femoral artery in a patient with Cushing disease: case report and literature review. Vascular 2009;17:163-167.

13) Fanelli F, Cannavale A, Gazzetti M, Fantozzi C, Taurino M, Speziale F. Spontaneous rupture of superficial femoral artery repaired with endovascular stent-grafting with use of rendez-vous technique, followed by delayed infection. Cardiovasc Intervent Radiol 2013;36:264-268.

14) Kakkos SK, Gohel M, Baekgaard N, Bauersachs R, Bellmunt-Montoya S, Black SA, et al. Editor's choice - European society for vascular surgery (ESVS) 2021 clinical practice guidelines on the management of venous thrombosis. Eur J Vasc Endovasc Surg 2021;61:9-82. 